\title{
INMUNONUTRICIÓN EN LA SALUD Y LA ENFERMEDAD
}

\author{
IMMUNONUTRITION IN HEALTH AND DISEASE
}

\author{
${ }^{1}$ Carlos Cuya Mamani
}

\begin{abstract}
RESUMEN
El sistema inmune cumple un rol esencial en la regulación de la homeostasis, junto con el sistema nervioso y el sistema endocrino, con formando un macrosistema regulador. La nutrición tiene enorme implicancia en la respuesta inmunitaria a los acentes lesivos, asi como en su inmunomodulación. El presente articulo describe la importancia que tienen los nutrientes en la programación del sistema inmunitario en las diferentes etapas de la vida, los cuales conducen a un estado óptimo de salud, especialmente en la gestación y en época infantil. Asimismo, se revisa las funciones que cumplen los nutrientes ante la respuesta inflamatoria en las diferentes enfermedades. especialmente en pacientes críticos y con cáncer, donde la inflamación se torna sumamente agresiva.
\end{abstract}

Palabras clave nutrición, sistema inmunitario

\section{ABSTRACT}

The immune system plays an essential role in the regulation of homeostasis, along with the nervous system and the endocrine system. forming a macro-regulator. Nutrition has huge implications in the immune response to harmful agents, as well as their immunomodulation. This article describes the importance of nutrients in the programming of the immune system at different stages of life, which lead to an optimal state of health, especially during pregnancy and childhood period. Also, the roles nutrients to the inflammatory response in various diseases, especially cancer patients critics and where inflammation becomes extremely aggressive is reviewed.

Keywords: nutrition, immune system.

\section{INTRODUCCIÓN}

Los organismos vivos, dentro de ellos nosotros los seres humanos, se caracterizan por mantener una bomeostasis ${ }^{\prime 2,}$, cs decir, un cquilibrio fisiológico que les permite mantener la vida. Tres son los sistemas homeostáticos por excelencia, que se encargan de ello: el sistema nervioso, el sistema endocrino y el sistema inmunitario. Este último, cuya función principal es defendernos de las infecciones y tumores desde el inicio de la vida, la cjerce en forma integrada, anatómica y funcionalmente, tanto con el sistema nervioso como con el sistema endocrino. De esta manera la homeostasis, y por ende la salud, se mantiene gracias a un macrosistema regulador: el neuroinmunoendocrino. Un ejemplo palpable de esta integración es el hecho comprobado ya, de cómo situaciones de ansiedad, depresión y estrés emocional se acompañan, muchas veces, de mayor susceptibilidad a padecer infecciones, cánceres y enfermedades autoinmunes.

Se denomina inmunonutrición a la capacidad que tienen los nutrientes de influir en la fisiología del sistema inmunc. En los seres humanos un buen estado nutricional repercute en un buen funcionamiento inmunológico y una buena salud. En personas desnutridas la respuesta inmunológica esta disminuida y ante un proceso de inflamación aguda y/o crónica el gasto energético y el estado nutricional sc alteran. La desnutrición afecta las diferentes barreras defensivas, tales como la piel y mucosas. También se alteran la inmunidad celular y humoral F.n la primera, ocurre una disminución en el tamaño del timo y alteración en la relación $\mathrm{CD} 8 / \mathrm{CD} 4$. En la inmunidad humoral se afectan los linfocitos $T$ cooperadores.

Es importante destacar, la importancia de los nutrientes con capacidad inmunomoduladora, tales como los antioxidantes y los ácidos grasos omega-3, los cuales son capaces de disminuir la activación del factor nuclear kappa beta (NFkB), que junto a la proteina activadora -1 , aumentan el ticmpo de respucsta inflamatoria, a nivel genético.

De esta manera, el estado nutricional de un ser humano repercute en el estado inmunológico. Ahora se invesuiga las implicancias de este conocimiento científico, cspecialmente la inmunomodulación que ejercen los nutrientes en las diferentes etapas de la vida, tanto en la salud como en la enfermedad. Han surgido nuevos conceptos que están relacionados a la inmunonutrición, tales como alimentos funcionales (aquellos que actúan en forma benćfica sobre una o más funciones del organismo, más allá de su efecto nutricional), probióticos ("microorganismos vivos" que, cuando se administran en cantidades adecuadas, confieren un bencficio a la salud del consumidor), prebióticos (glúcidos no digeribles, que estimulan el crecimiento o la actividad de los microorganismos autóctonos, resultando en un beneficio para la salud), simbióticos (mezclas de probióticos y prebióticos que generan una acción saludable sinérgica). ${ }^{5}$

\footnotetext{
Maestro en Medicina, Médico Cirujano General. Jefe del Departamento de Cirugía del Hospital III Yanahuara ESSALUD Arequipa. Docente de la Facultad de Medicina de la Univer sidad Nacional de San Agustín. Arequipa-Perú
} 


\section{FISIOLOGÍABÁSICADELSISTEMAINMUNITARIO}

Durante millones de evolución, el ser humano ha desarrollado un arsenal de defensas, el sistema inmunitario, que le permite responder a diferentes agentes invasivos; su accionar va desde simples barreras físicas (piel, mucosas), hasta células especializadas (leucocitos), además de otros componentes biológicos, químicos y nucleares (anticuerpos, citocinas y radicales libres). Los mecanismos de defensa frente a los microorganismos son de dos tipos, los cuales son interactivos, a saber: la inmunidad innata y la inmunidad adquirida.

La inmunidad innata está disponible desde el nacimiento, y constituye la primera defensa, Esencialmente son la piel, la secreción de las mucosas y la acidez del estómago. I a segunda barrera, la inmunidad adaptativa, se adquiere ya más tarde en la vida, luego de una infección que es vencida con éxito o luego de una vacunación. Tenemos una memoria inmunológica, guardada en grupos de linfocitos, produciéndose rápidamente anticucrpos en una siguiente infección. Disponemos de un sistema inmunitario humoral (linfocitos B), y otro celular (linfocitos T).

Los antigenos son las sustancias que nuestro organismo las reconoce como extrañas, produciendo respuestas inmunitarias específicas. Los linfocitos $\mathrm{T}$ y $\mathrm{B}$ se especializan en el timo y la médula ósea respectivamente, adquiriendo proteínas de superficie características, y de esta manera inmunocompetencia, es decir capacidad de producir respuestas inmunitarias ante estímulos apropiados. Algunas de dichas proteínas actúan como receptores de antigenos, que son moléculas capaces de reconocer antígenos específicos como CD4+ y CD8+. Fsta es la razón por la que los linfocitos T se llaman T4 y T8.

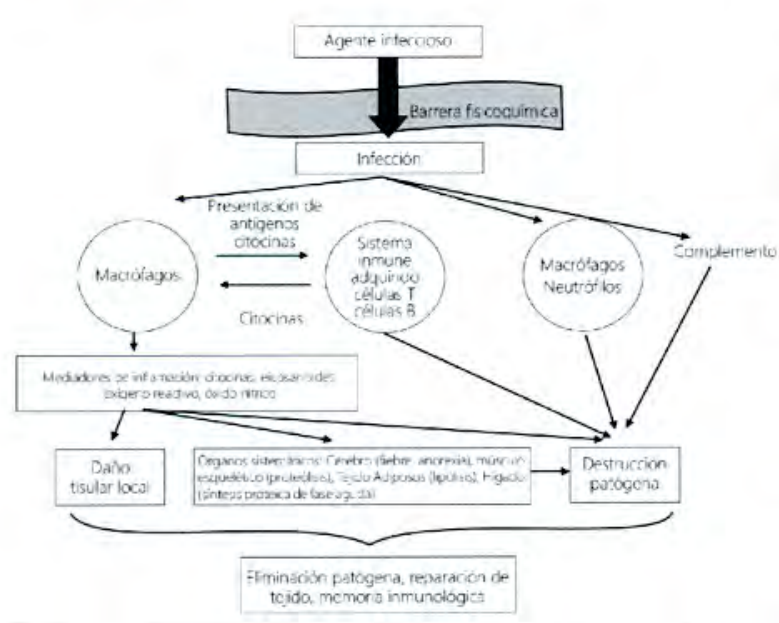

Figura 1. Panorama general de la respuesta inmunitaria.

Por otro lado, las citocinas son pequeñas hormonas proteicas que cumplen funciones, en el crecimiento y diferenciación celular, ya sea estimulando o inhibiéndolas. Fn suma, los linfocitos son células presentadoras de antígenos que producen citocinas. Hay un buen número de ellas, como el factor de necrosis tumoral alfa (TNF-a), y otras denominadas interleucinas (IL) como IL-1 o IL-6, llamadas tambićn pro- inflamatorias, porque se producen ante una lesión tisular de cualquicr tipo. Hay otras interleucinas, como $1 \mathrm{~L}-10 \mathrm{e} / \mathrm{L}-13$, que tienen efecto contrario. Es importante destacar que hay interleucinas que tienen funciones muy concretas.

Se ha desarrollado gran evidencia respecto a la polarización funcional de los linfocitos T4+, basados en la producción de diferentes tipos de citocinas. Concretamente, hay un grupo de linfocitos T4 helper de tipo 1 (Th1) que produce interferón gamma (INT- $\gamma), I L-2$ y factor de necrosis tumoral alfa (INF- $\beta$ ), los cuales activan macrófagos y cstán involucrados en la inmunidad celular. Fn el otro lado, los linfocitos T4 de tipo 2 (Th2) producen IL-4, IL-5, IL-10 e IL-13 median una intensa respuesta por anticuerpos e inhiben muchas acciones de los macrófagos.

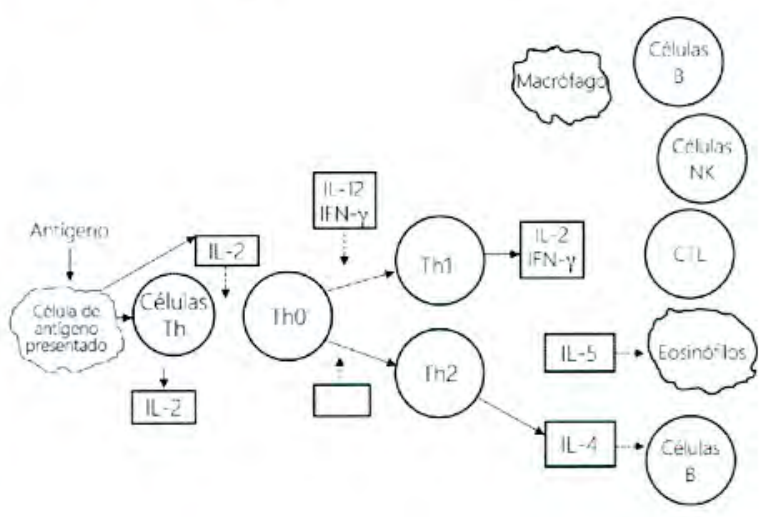

Figura 2. Funciones de los linfocitos T4 ayudadores.

\section{BASESFISIOLÓGICASDELAINMUNONUTRICIÓN}

El intestino es el órgano inmunitario más grande del organismo humano, de ahí la importancia, dada su función, de la inmunonutrición en la homeostasis. Dispone de más del $80 \%$ de linfocitos $\mathrm{B}$, que producen inmunoglobulinas y del $65 \%$ del tejido inmunitario. Tiene características peculiares respecto al resto del sistema inmune en general. Fl tejido linfático asociado al intestino ("gut association linfatic tisste": GALT), dispone de inmunocitos especializados, como linfocitos $\mathrm{T}$ intraepiteliales y células epiteliales presentadoras de antigenos, que no existen cn otros tejidos.

I. a respuesta inflamatoria que se produce en el intestino, debe ser localizada y regulada enteramente en el mismo. La exacerbación de la misma puede ser causa de inmunosupresión y esta está específicamente relacionada con linfocitos residentes totales bajos en las placas de Peycr, capa intraepitelial y lámina propia. Esta disminución de linfocitos se acompaña con alteración proporcional de células $\mathrm{T}$, promedio bajo de CD4 y CD8, y baja expresión de receptor de las células 'T y niveles reducidos de inmunoglobulina $\Lambda$ intestinal. Todo ello tiene como consecuencia una proliferación microbiana, lo cual lleva a un estado de inmunoparálisis, es decir a una incapacidad del sistema inmunitario para llevar a cabo sus funciones. Aquí es importante la contrarregulación de la respuesta inmunoinflamatoria que debe darse con la participación del sistema endocrino, nervioso e inmunológico que debe dar fin a la respuesta inflamatoria. 


\section{INMUNONUTRICIÓNEN LASALUD}

\section{Programación temprana del sistema inmune y su papel sobre la nutrición}

La investigación actual nos muestra cuan importantes son las atenciones que recibe un ser humano en los primeros años de vida, tanto cn el aspecto fisiológico y psicológico, que repercuten en su futuro, y hacen de él un ser saludable. Pero también, se están haciendo cada vez más importantes las interrclaciones gen-medioambiente que se dan en cl transcurso del embarazo, las cuales pueden ocasionar modificaciones permanentes en los procesos fisiológicos y la susceptibilidad a la enfermedad, alterando la expresión genética y la predisposición a la enfermedad mediante mecanismos epigenéticos. Prueba de cllo son cl aumento epidémico de patologías alérgicas y autoinmunes.

En humanos, se produce un desarrollo importante del sistema inmunitario ya en el útcro materno y también en las primeras semanas y meses luego del nacimiento, y ahí tiene una enorme importancia la nutrición. Estudios en modelos animales recientes nos muestran que la alteración de la dieta materna, a través de cambios epigenéticos en la expresión génica, pueden modificar el riesgo de enfermedad alérgica respiratoria en los descendientes. Hay además evidencias de que agentes medioambientales como los factores dictéticos, cl tabaco y la cxposición a bacterias, pueden modificar la respuesta inmunitaria del neonato.

Se han determinado propiedades inmunomoduladoras en muchos nutrientes de la dieta que parecen actuar a través de rutas metabólicas conocidas. Fntre estos nutrientes tencmos a los ácidos grasos poliinsaturados, antioxidantes y otras vitaminas. Se ha demostrado que la disminución en el consumo de ácidos grasos antiinflamatorios $n-3$, por la madre, los cuales se encuentran en el accite de pescado, origina un aumento de enfermedades alérgicas. Fstán en estudios otros nutrientes como la vitamina $\mathrm{D}$, vitamina $\mathrm{E}$ y el cinc. No debemos dejar de considerar otros factores como la exposición bacteriana materna (a productos patogénicos como no patogénicos), el entorno de citocinas en el que se encuentra el feto, $y$ hasta influencias endocrinas, pero todas ellas durante la gestación.

\section{Influencia de la leche humana en la salud del lactante}

La leche humana es la vía de comunicación entre el sistema inmunitario de la madre y el de su bebé. Así, el papel beneficioso de la lactancia materna sobre la función inmunitaria del recién nacido sc ha estudiado profusamente. Ya vemos, por lo pronto, la diferencia que tiene en la salud aquel niño que recibe lactancia materna del que no lo hace. El sistema inmunitario requicre maduración y esta se caracteriza por el desarrollo de una respuesta equilibrada Th1/Th2. La madurez de este sistema comprende también los mecanismos de regulación de la tolerancia y activación de la respuesta inmune. Si ellos no funcionan adecuadamente pueden propiciarse enfermedades como alergias alimentarias, problemas de autoinmunidad y enfermedades inflamatorias del intestino. ${ }^{10}$

La leche materna es una secreción inmunonutricional sui-géneris que contiene muchas moléculas de defensa (inmunoglobulina A secretora, lactoferrina y lisozima), citocinas (interleucina 8, resistente a las enzimas digestivas del lactantc), quimioquinas $\mathrm{y}$ factores de crecimiento. La regulación de los componentes de la leche humana están bajo el control de factores metabólicos, neuroinmunes y hormonales.

Como vemos, la leche humana tiene componentes que juegan un papel esencial en el desarrollo del sistema inmunitario del niño. Estos se encuentran en niveles bastante elevados, ya sea en el calostro o en la leche inicial, en comparación con los niveles encontrados en el plasma de la madre. Dependiendo de la fase y etapa de lactancia, también hay componentes celulares que están presentes en la leche materna, y estos son principalmente diversas variedades de leucocitos, y todos ellos se encuentran en forma activada.

Se ha encontrado que la leche materna no es estéril, al contrario es una fuente excelente de bacterias comensales para el intestino del recién nacido y una forma de defensa contra las infecciones. Las bacterias encontradas son los lactobacilos, estreptococos, estafilococos y enterococos. Fue a inicios de este siglo, que dos grupos europeos, de forma independiente, descubren la existencia de bacterias en la leche materna y su potencial probiótico.

El cquilibrio fisiológico del sistema inmunitario del recién nacido depende también de la colonización microbiana intestinal. Las bacterias intestinales propician las respuestas linfocitarias Th1, compensando cl desequilibrio Th2, típico de los recién nacidos.

\section{Micronutrientes y sistema inmunitario}

Las células que integran el sistema inmunitario, asi como otras que también participan en la respuesta inmunc, necesitan ser alimentadas, es decir, requieren de aporte óptimo de energía, macronutrientes y micronutrientes. Estas actúan en las diversas rutas metabólicas de la respuesta inmunológica. Hoy en día, se reconoce que el estado nutricional de una personal modula su inmunidad, por lo que es necesario suministrar los adecuados niveles de nutrientes para garantizar el adecuado funcionamiento del mismo.

Los micronutrientes tienen su función $\mathrm{en} \mathrm{cl}$ sistema inmunitario en tres niveles: 1 . reforzando las barreras fisicas (piel y mucosas), 2. en la inmunidad celular y 3. en la inmunidad humoral (producción de anticucrpos). Las vitaminas A, C, F. y el oligoelemento cinc ayudan a mejorar la función de la barrera cutánea. Las vitaminas $\mathrm{A}, \mathrm{B}_{6}, \mathrm{~B}_{12}, \mathrm{C}, \mathrm{D}, \mathrm{E}$ y los folatos, así como los oligoelementos hierro, cinc, cobre y selenio, actúan en conjunto para mejorar las actividades de defensa de las células inmunitarias. Además, todos cstos micronutrientes, excepto, la vitamina $\mathrm{C}$ y el hierro, son importantes en la producción de anticuerpos."

\section{Antioxidantes y sistema inmune}

Las biomoléculas son susceptibles de deterioro bajo el influjo de las especies reactivas de oxigeno (ROS), de los que los radicales libres son componentes; éstas se producen por la utilización de oxígeno en las vías metabólicas del organismo. De alli que se hace necesario un equilibrio entre oxidantes y sustancias antioxidantes para el mantenimiento de la salud, cl cual cobra mayor importancia $\mathrm{cn} \mathrm{cl}$ sistema inmunitario. Fl desequilibrio, es decir el estrés oxidativo, es la base de muchas enfermedades así como del envejecimiento. ${ }^{12}$ 
Los antioxidantes pueden ser: endógenos (generados en el organismo), y estos a su vez enzimáticos (supcróxido dismutasa, catalasa, glutatión peroxidasa, glutatión reductasa y las tiorredoxinas, y no enzimáticos (glutatión); también tenemos los antioxidantes exógenos (incorporados desde el exterior, generalmente en la dieta), entre ellos vitamina $\mathrm{C}$ o ácido ascórbico, vitamina $\mathrm{E}$ o tocofcroles, polifenoles y los carotenoides.

En diversos estudios, se ha demostrado la importancia de la ingestión de dictas o suplementos con más de un oxidante. Por ejemplo, la combinación de vitamina $\mathrm{C}$ y $\mathrm{E}$ ha resultado ser eficaz para disminuir la prevalencia e incidencia de la enfermedad de Alzheimer, aunque ello no se corroboró cuando se administró una sola de ellas. También se ha encontrado que la vitamina $\mathrm{C}, \mathrm{E}$ y el $\beta$-caroteno protegen los lipidos de las membranas celulares y neutralizan las especies reactivas de axigeno (ROS) y especies reactivas de nitrógeno (RNS), mediante acción cooperativa sinérgica, aún en pequeñas cantidades como las que se encuentran en la dieta.

\section{Ácidos grasos y sistema inmune}

Los ácidos grasos tienen funciones importantes en las células por ser una fuente importante de energía, son componentes esenciales de las membranas celulares, y también actúan como moléculas señalizadoras y reguladoras de la expresión génica. También se ha encontrado evidencias, desde hace tiempo, de que son capaces de regular la función inmune.

Por ejemplo, los ácidos grasos polïnsaturados omega-3, como el eicosapentanoico (EPA) y el docosabexanoico (DHA), han mostrado cfectos bencficiosos en una serie de enfermedades inflamatorias crónicas, como la arteriosclerosis y la obesidad. $^{13}$

\section{INMUNONUTRICIÓNENLAENFERMEDAD}

\section{Obesidad, sistema inmunitario y respuesta inflamato- ria}

En la actualidad estamos frente a una creciente explosión epidemiológica de enfermedades no transmisibles, entre ellas la obesidad, debido a un estilo de vida equivocado. Tanto el sobrepeso como la obcsidad sc caracterizan por un estado inflamatorio crónico de grado leve.

La inflamación es una poderosa respuesta de los tejidos vivos ante un agente lesivo, y es tan potencialmente destructiva que muchas veces puede tornarse inmanejable, como ocurre en la sepsis, cuando sc trata de una respuesta aguda, y que en el caso de enfermedades crónicas, juega un papel importante en las patologias cardiovasculares, cáncer, diabetes, asma e, inclusive, en la enfermedad de Alzheimer. Fl tejido adiposo es altamente sensible a la hipoxia, y la falta de oxígeno provoca respuesta inflamatoria con un aumento de la producción de señales cuyo objetivo esencial sería estimular la vascularización local.

El tejido adiposo es un órgano endocrino por su capacidad de producir hormonas como la leptina (factor adelgazante de la sangre); la adiponectina, la proteína más abundante secretada por $\mathrm{cl}$ adipocito y cuyas funciones más destacadas son la sensibilización a la insulina y una acción antiinflamatoria y de protección ante la lesión cardiovascu- lar. Otras proteinas que produce el tejido adiposo se agrupan bajo el nombre de adipoquinas, y sus funciones abarcan la regulación del metabolismo, presión sanguinea, inmunidad, hemostasia, angiogénesis o inflamación. ${ }^{14}$

Los adipocitos poseen también funciones semejantes a diversas células inmunitarias, como por ejemplo, producción de citocinas y activación del complemento. La concentración elevada de citocinas proinflamatorias (IL-6, IL$1 \beta$ y $T N F-a$ ), así como de algunas proteinas de la inflamación, se han relacionado con la masa grasa en adultos. Sc ha logrado evidenciar que los precursores de adipocitos tienen la capacidad de fagocitar y pueden convertirse en células parecidas a los macrófagos. Recientes estudios muestran que la acumulación de tejido graso origina la sobreexpresión de numerosos genes relacionados con la respuesta inmunitaria y la inflamación, lo que lleva a la producción de citocinas especialmente proinflamatorias. De esta manera, se hace ya comprensible como es que la obesidad cstá relacionada a múltiples enfermedades.

\section{Situación nutricional en pacientes infectados por VIH+ y con sida}

Los trastornos nutricionales están presentes en este tipo de pacientes, y los primeros estudios nos mostraron pérdida de peso y disminución de las proteínas corporales. El tratamicnto antirretroviral de alta cficacia ha propiciado una disminución de los niveles de desnutrición y una mejora de la supervivencia y en las funciones inmunológicas, aunque también tiene sus complicaciones como la lipodistrofia, que se relaciona a su vez con resistencia a la insulina y alteraciones en el metabolismo, tales como hipertrigliceridemia, intolerancia a la glucosa y diabetes. ${ }^{15}$

Actualmente, es evidente que la educación alimentaria y la asistencia nutricional son necesarias en los pacientes con SIDA. Además, se hace necesaria la evaluación bioquimica en esta enfermedad, la cual debe incluir, al menos, niveles séricos de albumina, transferrina, proteinas totales, colesterol, hemoglobina y hematocrito.

\section{Nutrición e inmunidad en el cáncer}

Un estilo de vida saludable y una dieta adecuada pueden prevenir del 30 a $40 \%$ de todos los cánceres según estudios epidemiológicos. Fs clara la evidencia científica que muestra que el exceso de peso corporal (sobrepeso, obesidad) es un factor de riesgo para el desarrollo y pronóstico de diversos cánceres como el de colon, mama, endometrio, riñón, csófago y próstata;

Son numerosos los estudios en los que se muestra la importancia que tiene la dieta y el estilo de vida (que incluye la actividad física) en la protección contra el desarrollo del cáncer, así como en el de las enfermedades crónicas. Tal protección puede ocurrir a través de acción directa en el sistema inmune (potenciándolo o suprimiéndolo); también sobre el desarrollo mismo del tumor, modulando la expresión genética, o por actividad antioxidantc.

La protección contra el desarrollo del cáncer se logra con una ingesta adecuada de vitaminas A y C, betacarotenos, selenio, cinc, probióticos y ácidos grados poliinsaturados n-3. Fstos modulan la respuesta inmunitaria o actúan como antioxidantes. Además, muchos componentes 
Cuya, C. Inmunonutrición en la salud y la enfermedad.

dietéticos pueden actuar como agentes quimiopreventivos, modulando la apoptosis o mucrte celular programada, interactuando a nivel celular o molecular, a través de la activación o inactivación selectiva de la expresión génica. Al respecto, es importante recordar que consumimos varios nutrientes en la dieta, $y$ estos tienen diferente acción en la expresión génica, por lo que resulta ideal hacer una adecuada combinación de nutrientes en la alimentación. Por ejemplo, tanto la ingesta baja de fibra y alta de carnes rojas, que origina un desequilibrio entre los ácidos grasos poliinsaturados n-3 y $\mathrm{n}-6$, han sido relacionados con un incremento en el riesgo de cáncer. ${ }^{16}$

Respecto al tratamiento mismo del cáncer, la investigación cientifica también muestra datos favorables, respecto a la inmunonutrición, en la potenciación del sistema inmune luego del tratamiento quirúrgico. Esta se lleva a cabo con la administración de ácidos grasos poliinsaturados $\mathrm{n}-3$, arginina y nucleótidos. Además, ha resultado esencial el uso de la inmunonutrición preoperatoria, la cual mejora el resultado del tratamiento quirúrgico del cáncer del tracto gastrointestinal superior, reduciendo el número de complicaciones.

\section{Inmunonutrición en pacientes quirúrgicos y en estado crítico}

Los traumatismos, las quemaduras, las heridas y la cirugía producen una respuesta inflamatoria que puede llegar a ser inmanejable y perjudicial en algunos pacientes. FI nombre que toma esta respuesta inflamatoria es el síndrome de respuesta inflamatoria sistémica (SIRS, sistemic inflamatory response syndrome) y su característica principal es que produce niveles excesivos de citocinas inflamatorias, especialmente factor de necrosis tumoral alfa (TNF- $\alpha$ ), interleucina 1 beta (IL-1 $\beta)$, interleucina 6 (IL-6) e interleucina 8 (IL-8). A esta hiperinflamación le pucde scguir un estado de inmunosupresión, en el cual se incrementa la susceptibilidad a la infección y luego un estado de sepsis y shock séptico, que se conocen como sindromes sépticos y son causa de muerte en pacientes gravemente enfermos.

En estos casos, la inmunonutrición tiene que ver con la administración de nutrientes específicos o su combinación de éstos en este tipo de pacientes. Generalmente los afectados suclen recibir nutrición artificial vía parenteral o enteral. I a idea básica es que los nutrientes pueden mejorar las respuestas inmunológicas mediadas por células en forma significativa. Desde el punto de vista de nutrición artificial, este concepto se amplia para incluir modificación de los procesos hipcrinflamatorios (que incluyc el estrés oxidativo) y la mejora de la función intestinal con la finalidad de prevenir la translocación bacteriana.

Es importante tener en cuenta que la nutrición artificial, al igual que la natural, tiene como fundamento el consumo de macronutrientes (proteinas, carbohidratos, lípidos) y también micronutrientes (vitaminas y minerales). La nutrición artificial contiene nutrientes añadidos o cantidades más elevadas de nutrientes que los que están presentes en la dieta habitual. Los nutrientes más importantes comprenden: glutamina, arginina, $\mathrm{N}$-acetilcistcína, aminoácidos de cadena ramificada, nucleótidos, ácidos grasos de cadena larga n-3, vitaminas antioxidantes, elementos traza, taurina.

\section{REFERENCIAS BIBLIOGRÁFICAS}

1. Dvorkin M, Cardinali D, Iermoli R. Bases Fisiológicas de la Práctica Médica 14ava ed. Editorial Médica Panamericana 2010.

2. Mezquita C. Fisiología Médica. Del razonamiento fisiológico al razonamiento clínico $1 \mathrm{ra}$. ed. Editorial Médica Panamericana 2011.

3. Campillo J. El mono estresado 1ra. ed. Drakontos 2012.

4. Gardner D, Shoback D. Endocrinología básica y clínica 9a. ed. McGraw Hill 2012.

5. Suárez J. Microbiota autóctona, probióticos, prebióticos. Nutr H losp. 2015; 31(Supl. 1):3-9.

6. Crabtree U. Inmunonutrición. Revista Gastrohnup Año 2010 Volumen 12 Número 3:113-119.

7. Abbas $\Lambda$, Tichtman $\Lambda$, Pillai S. Inmunología celular y molecular 7 ma.ed. Elsevier 2012.

8. Feldman M, Friedman L, Brandt L. Enfermedades digestivas y hepáticas. Fisiopatología, diagnóstico y tratamiento 8va. ed. Elsevier 2012.

9. Prescott S. Implicación de los acontecimientos in útero en el desarrollo de las enfermedades alérgicas. En: Inmunonutrición en la salud y enfermedad 1 ra. ed. Editorial Médica Panamericana 2011.

10. Mujico J, Marcos A. Factores inmunológicos de la leche materna. En: Inmunonutrición en la salud y enfermedad 1 ra. ed. Fditorial Médica Panamericana 2011.

11. Maggini S, Beveridge S. Vitaminas y oligoclementos que mejoran la función inmunitaria mediante el refuerzo de las barreras epiteliales y las respuestas celular y humoral En: Inmunonutrición $\mathrm{cn}$ la salud y enfermedad $1 \mathrm{ra}$. ed. F.ditorial Médica Panamericana 2011.

12. De la Fuente M. Los antioxidantes y la función inmunitaria. En: Inmunonutrición en la salud y enfermedad $1 \mathrm{ra}$ ed. Fditorial Médica Panamericana 2011.

13. Yaqoob P. Efecto regulador de los ácidos grasos poliinsaturados n-3: mecanismos responsables. En: Inmunonutrición en la salud y enfermedad 1ra. ed. Editorial Médica Panamericana 2011.

14. Warnberg J, Romeo J, Marcos a. Obesidad en inflamación en el adolescente. En: Inmunonutrición en la saludy enfermedad, 1 ra. ed. Lditorial Médica Panamericana 2011.

15. Slobodianik N, Stambullian M, Feliu M. Situación nutricional en pacientes infectados con VIH y con SIDA. Lin: Inmunonutrición en la salud y enfermedad, 1ra. ed. Editorial Médica Panamericana 2011.

16. Valdés-Ramos $R$, Beníte\%-Arciniega $\Lambda$. Nutrición $e$ inmunidad en cáncer. En: Inmunonutrición en la salud y enfermedad, 1ra. ed. Editorial Médica Panamericana 2011.

17. Calder P. Inmunonutrición en pacientes quirúrgicos y en estado crítico. En: Inmunonutrición en la salud y enfermedad, 1ra. ed. Editorial Médica Panamericana 2011. 